\title{
A Method for Reducing Time-to-Solution in Quantum Annealing Through Pausing
}

\author{
Michael R. Zielewski \\ michael@hpc.is.tohoku.ac.jp \\ Graduate School of Information Sciences \\ Tohoku University \\ Sendai, Japan
}

\author{
Hiroyuki Takizawa \\ takizawa@tohoku.ac.jp \\ Cyberscience Center \\ Tohoku University \\ Sendai, Japan
}

\begin{abstract}
Recent research has shown that alternative annealing schedules provide the means for improving performance in modern quantum annealing devices. One such type of schedule is forward annealing with a pause, in which there is a period of time when system evolution is paused. While the results from using this type of schedule have been promising, effectively using a pause is not a trivial task. One challenge associated with introducing a pause into the schedule is determining the point in the anneal at which the pause will start. Additionally, tuning the schedule in real-time requires a significant amount of time. A second challenge is that while a pause may increase the number of correct solutions returned from the annealer, the time-to-solution, a standard metric for measuring performance in quantum annealing, will not necessarily be improved. We propose a method for constructing annealing schedules containing a pause that avoids the costly process of determining the optimal pause location in an online manner. We also evaluate our method on the subset sum problem, a problem of practical significance, and show that our method is able to achieve a $70 \%$ reduction in time-to-solution from a standard schedule containing no pause.
\end{abstract}

\section{CCS CONCEPTS}

- Hardware $\rightarrow$ Quantum computation; • Computer systems organization $\rightarrow$ Quantum computing.

\section{KEYWORDS}

quantum annealing, D-Wave, annealing schedule, pausing

\section{ACM Reference Format:}

Michael R. Zielewski and Hiroyuki Takizawa. 2022. A Method for Reducing Time-to-Solution in Quantum Annealing Through Pausing. In International Conference on High Performance Computing in Asia-Pacific Region (HPC Asia2022), January 12-14, 2022, Virtual Event, fapan. ACM, New York, NY, USA, 9 pages. https://doi.org/10.1145/3492805.3492815

\section{INTRODUCTION}

The field of quantum computing is experiencing a surge in interest due to the increasing availability of quantum computing devices. However, not many devices contain sufficient qubits to

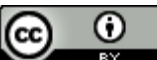

This work is licensed under a Creative Commons Attribution International 4.0 License.

HPC Asia2022, January 12-14, 2022, Virtual Event, Japan

(c) 2022 Copyright held by the owner/author(s).

ACM ISBN 978-1-4503-8498-8/22/01.

https://doi.org/10.1145/3492805.3492815 solve problems of practical size. One group of quantum computers that stands apart are those manufactured by D-Wave Systems [8]. These quantum computers contain significantly more qubits than other quantum computers, with the latest generation containing more than 5,000 qubits [3]. This large number of qubits enables DWave quantum computers to solve problems of non-trivial sizes in various applications, including scheduling, traffic flow, and clustering $[18,22,26]$. Another factor that differentiates D-Wave quantum computers from most others is that D-Wave quantum computers implement quantum annealing.

Quantum annealing is a metaheuristic for optimizing problems of binary variables through quantum fluctuations. Initially, quantum annealers are prepared in a state in which quantum fluctuations are used to explore the state space. The target problem is then slowly introduced, and becomes dominant. At the end of the anneal, the system is in a low energy state, close to the optimal solution of the target problem.

The probability with which the annealer produces an optimal solution is influenced by many factors, including problem and hardware properties. Recently, using annealing controls to modify annealing schedules has been attracting attention as a method for improving the quality of solutions returned by quantum annealers [5, 14-16, 23, 25, 36]. While many controls have been available only in the latest two generations of quantum annealers, and thus subject to limited testing, results using these controls to modify annealing schedules are promising. In many cases, correctly utilizing annealing controls leads to higher quality results, or more valid solutions.

We focus on annealing schedules that include a pause, during which evolution of the system Hamiltonian temporarily ceases. Previous works have shown that pausing enables thermalization, leading to orders of magnitude improvement in ground state probability [25] or a potential reduction in the time required to find a solution [15]. However, these results also make it clear that in order for a pause to be effective, it must be placed at or near an instance-wise optimal location. When a pause is placed far from the optimal location, no increase in performance will be observed. Determining this location typically involves examining empirical results from annealing with pauses at various locations, as numerical methods are intractable for systems of practical size. Thus, the process of finding the optimal location requires many anneals and increases the time required to find a solution. Furthermore, when solving many problems, this process must be repeated and a considerable amount of annealing time is required. This leads to simpler schedules without a pause being superior in both efficiency and speed. 
Motivated by the promising results from pausing, and considering the challenges associated with determining optimal pause locations, we propose a method that is able to reduce solution time, mitigate the impact of the time required for determining optimal pause locations, and limit the amount of time required to do so. Our proposal consists of two main stages. In the first, we focus on profiling a problem class and determining an optimal annealing schedule with a pause. We use empirical results from trials with various configurations to accomplish this. The second stage of our proposal presents a method of applying the previously found optimal schedule in a real-time manner such that an improvement in solution time over a baseline schedule that lacks a pause is achieved. We evaluate our proposal on an NP-complete problem, the subset sum problem, and show that our method is able to produce a schedule that results in a 3.4 times speedup over the baseline.

The rest of this work is organized as follows. We first present additional background in Section 2. Then, in Section 3, we propose the method used in this work for determining the optimal annealing schedule with a pause for a given problem. We also introduce the problem and formulation used in our evaluation. Our evaluation setup as well as the results from our evaluation are presented in Section 4. In Section 5, we provide a brief review of related work and clearly identify the area that our work advances. We give our conclusions in Section 6 and provide what we consider to be promising future directions for this work.

\section{BACKGROUND}

In this section, we provide the necessary background on both quantum annealing and annealing schedule modifications.

\subsection{Quantum Annealing}

Quantum annealing is an optimization algorithm in which diminishing quantum fluctuations are used to explore a system's state space and find the system's minimum energy state [19]. While quantum fluctuations are being reduced, the system gradually evolves towards the problem to be solved. System evolution is modeled by the Hamiltonian

$$
H(s)=A(s) H_{\mathrm{D}}+B(s) H_{\mathrm{P}},
$$

where $H_{\mathrm{D}}$ is the driver Hamiltonian that introduces quantum fluctuations, and $H_{\mathrm{P}}$ is the problem Hamiltonian encoding the problem to be solved. The systems evolve from time $t=0$ to the total run time $t_{\mathrm{t}}$. The strengths of the driver and problem Hamiltonians are governed by the time-dependent functions $A(s)$ and $B(s)$, respectively, where the annealing fraction $s$ ranges from 0 to 1 . The problem Hamiltonian is in the form of an Ising model given by

$$
H_{\mathrm{P}}=\sum_{i}^{N} h_{i} s_{i}+\sum_{i<j}^{N} J_{i, j} s_{i} s_{j},
$$

where for $N$ spin variables $\left[s_{1}, \ldots, s_{N}\right] \in\{-1,1\}, h$ defines the linear biases and $J$ defines the quadratic couplings. An equivalent formulation that may be more frequently used in optimization is the quadratic unconstrained binary optimization (QUBO) problem. Using this formulation, the problem Hamiltonian is given by

$$
H_{\mathrm{P}}=x^{\top} Q x
$$

in which binary variables $\left[x_{1}, \ldots, x_{N}\right] \in\{0,1\}$ are used and the linear and quadratic terms are stored in the diagonal and off-diagonal components of an upper diagonal matrix $Q$. We will use QUBO form for the remainder of this work.

Solving optimization problems with quantum annealing requires two preprocessing steps, problem formulation and embedding. First, in problem formulation, a natural problem is converted to QUBO form. Primarily, this involves creating an objective function to be minimized. However, optimization problems are also often subject to various constraints. These constraints are typically modeled as penalties to the objective function. Following problem formulation is embedding, which maps the variables of the QUBO problem to qubits on the physical annealer. This step is necessary because qubits on the annealer have limited connectivity, and certain logical variables in the QUBO problem may need to be represented by a "chain" of multiple physical qubits in order to satisfy QUBO problem connectivity. While there are problems that match the structure of the annealer and do not require chains, termed native problems, many problems derived from real-world applications have complex structures requiring long chains. In general, chain and embedding size should be minimized for performance reasons [9], but the computational complexity of embedding algorithms makes this impractical. These circumstances introduce a time-performance trade-off before problems have been submitted to the annealer [36].

\subsection{Annealing Schedules}

Following the required preprocessing for annealing, an annealing schedule that determines the values of $A$ and $B$ throughout the anneal must be constructed. The most standard type of annealing schedule is forward annealing in which $s(t)=\frac{t}{t_{\mathrm{t}}}$. A forward annealing schedule with values representative of the system used in this work is shown in Figure 1 [2]. While control over the schedule was limited in earlier generations of D-Wave devices, the latest devices provide controls to modify system evolution. One specific modification that these controls enable is the inclusion of a pause in the annealing schedule, where a pause is defined as a period of time in which $s$, and thus $A$ and $B$, are held constant. Defining a schedule with a pause requires that a pause location $s_{\mathrm{p}}$ be defined. Additionally, $t_{\mathrm{t}}$ must be composed of the sum of two component times: the annealing duration $t_{\mathrm{a}}$, and the pause duration $t_{\mathrm{p}}$. Note that in forward annealing $t_{\mathrm{p}}=0$, and thus $t_{\mathrm{t}}=t_{\mathrm{a}}$. The annealing fraction for a schedule with a pause is then defined as

$$
s(t)= \begin{cases}\frac{t}{t_{\mathrm{a}}} & t \leq t_{\mathrm{a}} \cdot s_{\mathrm{p}} \\ s_{\mathrm{p}} & t_{\mathrm{a}} \cdot s_{\mathrm{p}}<t \leq t_{\mathrm{a}} \cdot s_{\mathrm{p}}+t_{\mathrm{p}} \\ \frac{t-t_{\mathrm{p}}}{t_{\mathrm{a}}} & t_{\mathrm{a}} \cdot s_{\mathrm{p}}+t_{\mathrm{p}}<t .\end{cases}
$$

Figure 2 shows annealing fraction values for a standard forward anneal of $1 \mu \mathrm{s}$ and a forward anneal of $1 \mu \mathrm{s}$ with a $1 \mu$ s pause inserted at $s=0.5$. From this figure it is clear to see that when both types of schedules have equal annealing durations, the rate at which $s$ evolves is the same except at the pause location.

\subsection{Performance Metrics}

The main motivation behind modifying annealing schedules is improving performance. Using the controls available to current quantum annealers, many works have shown the ability of modified 


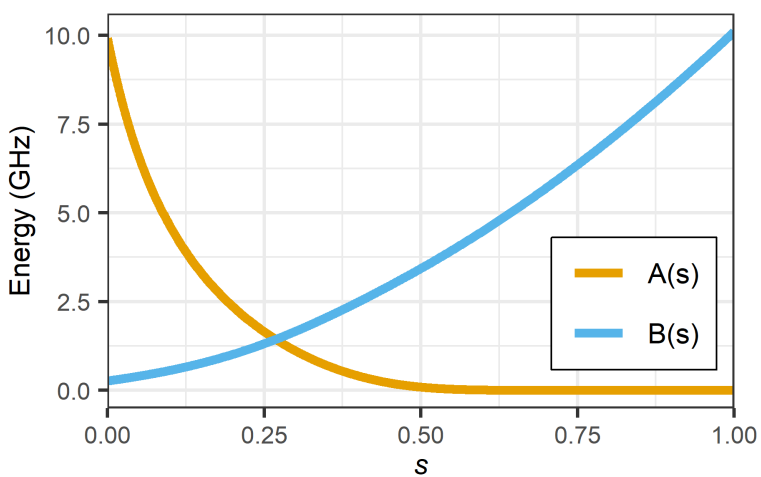

Figure 1: A forward annealing schedule.

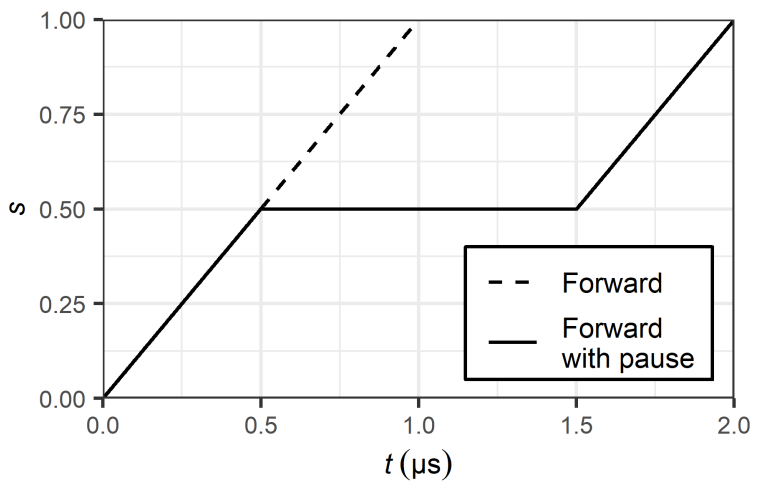

Figure 2: Values of the annealing fraction $s$ for a forward annealing schedule and a forward annealing schedule containing a pause. In both schedules $t_{\mathrm{a}}=1 \mu \mathrm{s}$. For the schedule containing a pause, $t_{\mathrm{p}}=1 \mu$ s and $s_{\mathrm{p}}=0.5$.

annealing schedules to achieve this [14-16, 18, 23, 25, 32, 35, 36]. One typical performance metric used in these works is the empirical probability of solving a problem, calculated as

$$
P_{\text {solve }}=\frac{n_{\text {solve }}}{n_{\mathrm{a}}},
$$

where $n_{\text {solve }}$ is the number of configurations from the annealer that solve the problem, and $n_{\mathrm{a}}$ is the total number of anneals performed. While this metric is suitable for comparing some schedules, it is unsuitable for comparing schedules where $t_{\mathrm{t}}$ is not equal. The reason for this is that the metric cannot capture the relationship between total annealing time and the time required to solve a problem.

A second common metric is time-to-solution (TTS), which measures the time required to obtain a solution with a certain probability, typically specified as 0.99 . TTS is calculated as

$$
\frac{\log (1-0.99)}{\log \left(1-P_{\text {solve }}\right)} \cdot t_{\mathrm{t}}
$$

TTS can be used to compare schedules of different lengths and determine which will produce a correct solution in less time. This metric is especially useful for comparing schedules where an increase in $P_{\text {solve }}$ does not necessarily reduce the time required to solve a problem. An additional benefit of this metric is that it can be used to compare results across quantum and classical solvers. By examining both $P_{\text {solve }}$ and TTS, one can determine both an optimal schedule and the point past which longer pauses result in diminishing returns.

In this work, we focus on schedules that utilize a pause to improve $P_{\text {solve }}$. Marshall et al. [25] provide theoretical background as to why pausing can improve $P_{\text {solve }}$, as well as supporting experimental results. In summary, a system will experience a transfer of population from low energy states to higher energy states in the area around the system's minimum gap, but a pause placed shortly after this location may increase thermalization, thus leading to an increase in $P_{\text {solve }}$. Furthermore, for each problem instance, there is an optimal pause location, which we denote by $s_{\mathrm{p}}^{\mathrm{opt}}$. Though not the main focus, their work also reveals one of the main challenges associated with annealing with a pause; estimating the optimal pause location from the minimum gap is intractable for all but small systems, and determining the location empirically requires a significant number of anneals. Performing either method in realtime expecting to observe a reduction in TTS is unwise, as the time required to do so is much greater than the TTS of a simpler schedule without a pause.

To justify this claim, we use an additional metric repetitions-tosolution (RTS) with probability 0.99 . RTS is calculated as

$$
\frac{\log (1-0.99)}{\log \left(1-P_{\text {solve }}\right)}
$$

Note that RTS and TTS are closely related and that TTS is simply RTS accounting for the time of each anneal, i.e., TTS $=\mathrm{RTS} \cdot t_{\mathrm{t}}$. Consider a scenario in which the optimal pause location is being estimated in real time in order to provide an improvement in TTS. We define a baseline as a forward annealing schedule with no pause. For a schedule that includes a pause located far from the true optimal pause location, the expected $P_{\text {solve }}$ is approximately the same as the baseline, and thus the RTS will also be the same. However, due to the increased time required for the schedule with the pause, its TTS will be greater than that of the baseline. To account for this and determine the maximum number of anneals in which the modified schedule must improve upon the baseline, the baseline RTS can be divided by the factor representing the increase in run time over the baseline, $\frac{t_{\mathrm{a}}+t_{\mathrm{p}}}{t_{\mathrm{a}}}$. In summary, as the pause duration increases, a schedule with a pause must improve upon the baseline $P_{\text {solve }}$ in fewer anneals in order to improve TTS.

The significance of this becomes apparent when estimating the optimal pause location from empirical data. Due to the probabilistic nature of the annealer, repeated anneals using the same schedule will return different results. Thus, the performance of a schedule cannot be estimated from a single anneal. In order to robustly estimate a schedule's performance, a single annealing run consisting of many anneals is performed. Therefore, as pause duration increases, estimation of the optimal pause location must be accomplished in a diminishing number of annealing runs.

To emphasize the challenge in achieving an improvement in TTS while estimating the optimal pause location in real-time, we 


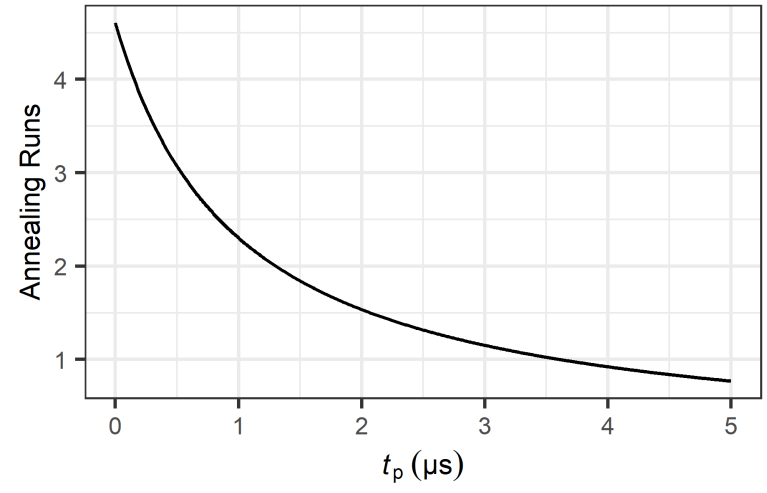

Figure 3: The maximum number of annealing runs for tuning a schedule with a pause to achieve a higher TTS than a baseline schedule with no pause, as a function of pause duration. $t_{\mathrm{a}}=1 \mu \mathrm{s}$. Values for $t_{\mathrm{p}}$ are selected to include the range of values tested in this work.

consider the case in which the $P_{\text {solve }}$ for a baseline schedule is nonzero, but minimized. This leads to TTS being maximized, which also maximizes the number of annealing runs within which estimation of an effective schedule with a pause must be completed. The baseline schedule we select is forward annealing with minimum possible annealing time, which has previously been shown to lead to a solution in the shortest time [6,28]. For the annealers used in this work, this time is $1 \mu \mathrm{s}$. Then, we assume that for an annealing run consisting of many anneals (typically $n_{\mathrm{a}}>1 \times 10^{3}$ in practice) the minimized value that $P_{\text {solve }}$ takes is $\frac{1}{n_{\mathrm{a}}}$. Following these assumptions, the number of annealing runs $\frac{\text { RTS }}{n_{\mathrm{a}}}$ for the baseline to find a solution can be simplified as $-\log (1-0.99)$. Figure 3 shows the number of annealing runs within which real-time estimation of the optimal pause location must be successful in order to achieve a reduction in TTS over the baseline. This figure clearly shows the relationship between pause duration and annealing runs; as pause duration increases, an improvement in $P_{\text {solve }}$ must be achieved in fewer annealing runs in order for real-time tuning of the optimal pause location to be effective. If no improvement is made, it would be more effective to anneal with the baseline schedule. For short pauses, an improvement in TTS must be achieved in very few annealing runs. This is challenging because there is no prior knowledge about the optimal pause location, the area in which pausing will be effective is small, and the improvement in $P_{\text {solve }}$ will also be small. However, for longer pauses where the improvement in $P_{\text {solve }}$ is greater and the area in which pausing is effective is larger, even fewer annealing runs are available. Therefore, we conclude that consistently achieving an improvement in TTS while tuning a schedule in real-time is not feasible.

\section{A METHOD FOR EFFICIENTLY AND EFFECTIVELY UTILIZING PAUSING}

Previous works have shown that pausing can be used with embedded problems to improve $P_{\text {solve }}$ and even achieve a speedup in TTS when compared to a standard forward annealing schedule $[15,36]$.
However, it is not yet clear how an improvement in TTS can be attained when accounting for the time required to find an optimal annealing schedule with a pause in real-time. In this section, we present our method for achieving a reduction in TTS through pausing while minimizing the impact of the process of finding an optimal schedule.

In Section 2, we established a baseline schedule for solving a problem. We also showed that real-time tuning of a schedule with a pause will result in a worse TTS. Now, we introduce core assumptions of our method that allow it to improve upon the baseline schedule. We assume that a given problem to be solved can be decomposed into similarly structured sub-problems without significant overhead, and that a solution to the original problem can be formed from solutions to the sub-problems. Combined with the observation that similar problems have similar optimal pause locations $[15,25]$, this assumption allows us to profile the sub-problems, and determine an optimal schedule. This schedule can then be reused for future anneals on problems belonging to the same class, where a class is defined as a collection of problems that have similar structure and parameters. While the schedule may be sub-optimal at an instance-wise or sub-problem level, it will be effective for a class of problems as a whole. We consider this problem requirement to be acceptable, as such types of problems that are suitable for decomposition are not uncommon in real-world applications. For example, Abbott et al. [4] present the problem of maximizing data transfer in a wireless network and show its decomposition into similar sub-problems.

We present a second application that is amenable to decomposition. Originally introduced by Caprara et al. [10], consider the task of minimizing waste when allocating raw materials for products. Such a problem can be decomposed into sub-problems that minimize the waste of one shipment of raw material, and can be modeled by the subset sum problem (SSP). The SSP is defined as follows. Given a set of integers $I$ and a target sum $C$, find $x$ such that

$$
\sum_{i=1}^{N} x_{i} I_{i}=C,
$$

where $x_{i} \in\{0,1\}$. In the application presented by Caprara, the amount of raw material required for a product is represented by the integers in the SSP, and thus an optimal selection of products that results in no waste is modeled by Equation 8. In the case where zero waste is impossible, then waste should be minimized. This task can be easily formulated for quantum annealing. The corresponding QUBO formulation is constructed from the squared difference between $C$ and the sum of selected integers, which can be considered a penalty and given by

$$
\left(C-\sum_{i=1}^{N} x_{i} I_{i}\right)^{2}
$$

A solution that satisfies Equation (8) will result in a value of zero for Equation (9), and thus represent a solution to the sub-problem. Additionally, solving each of the sub-problems directly solves the original problem of minimizing the total waste among all shipments. While we have presented two specific applications that can 
be used with our method, any problem that can be decomposed similarly provides our method with opportunities to utilize a class-wise optimal schedule and reduce TTS.

We now propose a method for achieving an improvement in TTS through pausing, over a baseline schedule with no pause, that can be applied in real-time. Our proposal consists of the following steps:

(1) Anneal with schedules parameterized by various pause durations and locations.

(2) Aggregate results and evaluate schedules.

(3) Determine a class-wise optimal schedule.

(4) Anneal with the estimated optimal schedule.

Our method can be divided into two stages: in the first stage composed of the first three steps, we estimate an optimal schedule in an offline manner, while in the final stage corresponding to the last step, we apply the schedule to reduce TTS.

In the first step of our proposal, we collect sufficient data to determine where a problem instance's optimal pause location lies. Many observations may be required in order to differentiate between an improvement in $P_{\text {solve }}$ due to noise, and a true improvement in $P_{\text {solve }}$ due to pausing, especially for shorter pauses for which the improvement is expected to be small. Thus, we will use schedules with pauses at various locations, and anneal many times at each location. We repeat this process for multiple pause durations and problem instances, so that in later steps we can select the optimal combination of parameters that leads to the shortest TTS.

In the second step of our proposal, we find the most effective schedules. We first group our results by problem instance and pause duration. Then, we summarize each group of results by selecting the schedule that resulted in the greatest $P_{\text {solve }}$. The set of results produced from this process represents the most effective schedules tested, and thus also the instance-wise optimal pause locations. From this, we can compute various features of interest, including the dispersion of optimal pause locations and the most common optimal pause location.

We estimate the class-wise optimal schedule in the third step of our proposal. Using the optimal pause locations from the previous step, we find the median optimal pause location for each pause duration. We consider these combinations to be the optimal schedule for each pause duration. Then, using the data from the first step, we collect the TTS values corresponding to these optimal schedules. In some cases, $P_{\text {solve }}=0$, which would lead to a TTS of infinity. In order to provide a more comprehensive comparison of TTS for different schedules, we set $P_{\text {solve }}$ to take a minimum value of $\frac{1}{n_{\mathrm{a}}}$ when it would otherwise be zero, for Equation (6) only. Note that unless a schedule with a pause can significantly improve upon one without a pause, this would naturally lead to shorter schedules without a pause resulting in the lowest TTS. Thus, we consider this modification to be acceptable. We then sum the TTS values by pause duration. Finally, we estimate the class-wise optimal schedule as the one that results in the shortest TTS. Note that since our goal is to select a schedule that minimizes TTS for future problems, the time required to determine the schedules is not included in the sum. At the end of this step, we have a single annealing schedule that can be used for future anneals.
Lastly, in the fourth step of our proposal, we use the estimated class-wise optimal schedule found in the previous step to achieve a reduction in TTS. Since an optimal schedule has already been estimated, there is no need to evaluate multiple schedules; thus, no time is spent on further tuning the schedule. This enables our method to reduce TTS in real-time, provided that the schedule used is effective. In our evaluation, we simulate real-time application of our method by using unique and unseen problem instances for testing. To ensure the effectiveness of our method, we anneal with the schedule found to be optimal for each pause duration. We then group our results by pause duration, sum the TTS values as done in Step 3, and use the speedup over the baseline to empirically show the effectiveness of our method.

Our proposal is simple and straightforward, yet capable of providing a significant improvement in $P_{\text {solve }}$ and TTS. Additionally, our method is not limited to specific types of problems, and is therefore promising for widespread application.

\section{EVALUATION AND RESULTS}

In this section, we detail our evaluation parameters and provide the results from our proposed method.

\subsection{Evaluation Setup}

Our evaluation will be carried out on a D-Wave Advantage system [3], the latest generation of D-Wave quantum annealers that is available. We use the SSP for our evaluation, using the problem formulation presented in Section 3. The logical graph of the resulting QUBO problem is fully connected. To take advantage of the increased connectivity and number of qubits of the annealer, we generate SSP instances of a size that is unable to be solved directly on previous generation hardware. We set $N=70$, select $I_{i}$ uniformly from $[1,50]$, and set $C=\left\lfloor\frac{1}{2} \sum_{i=1}^{N} I_{i}\right\rfloor$. We use a clique embedding method [1] to generate an embedding with uniform chain lengths. Note that since problem instances are fully connected and contain the same amount of nodes, the embedding can be generated in an offline manner and cached to avoid lengthy online recomputation.

For our evaluation, we generate 100 unique SSP instances. To avoid biased results from evaluating our method on the same problem instances that were used to determine a class-wise optimal pause location, we partition the generated instances into two sets: the first set of 20 instances is used for steps 1 to 3 of our proposal, and the second set of 80 instances is used for Step 4. For determining a class-wise optimal pause location, annealing schedules are generated with $t_{\mathrm{a}}=1 \mu \mathrm{s}, t_{\mathrm{p}} \in\{0.1,0.3,0.6,1,5\} \mu \mathrm{s}$, and $s_{\mathrm{p}} \in[0.2,0.6]$ at intervals of 0.01 . Our selection of pause durations is intended to cover short pauses, for which pausing is expected to yield an improvement in $P_{\text {solve }}$ and TTS, and longer pauses, for which a greater improvement in $P_{\text {solve }}$ is expected yet an increase in TTS may not occur. Each annealing run consists of $n_{\mathrm{a}}=10,000$ anneals. We use gauge transformations, a technique commonly used to decrease the effects of hardware biases in annealing and produce less noisy results [15], and set the number of transformations to 50. When results are returned from the annealer, each chain of qubit values must be converted to a single value for the variable that the chain represents. However, qubit values within a chain do not always 
Table 1: Estimated optimal pause locations and Sum of TTS

\begin{tabular}{ccc}
\hline$t_{\mathrm{p}}(\mu \mathrm{s})$ & $s_{\mathrm{p}}^{\mathrm{opt}}$ & Sum of TTS $(\mu \mathrm{s})$ \\
\hline 0.1 & 0.375 & 846687 \\
0.3 & 0.410 & 491992 \\
0.6 & 0.430 & 303513 \\
1.0 & 0.430 & 299845 \\
5.0 & 0.465 & 322124 \\
\hline
\end{tabular}

agree. In the case that the values of a chain are not consistent, we consider the individual configuration incorrect.

\subsection{Evaluation Results}

We first present our results for the first stage of our proposal in which our goal is to estimate a class-wise optimal schedule for the SSP and parameters used to generate instances. Figure 4 shows $P_{\text {solve }}$ for a range of values of $s_{\mathrm{p}}$ and $t_{\mathrm{p}}$. The patterns in the data match other works; as the pause becomes longer, the maximum improvement in $P_{\text {solve }}$ increases, the area in which pausing is effective becomes larger, and the optimal pause location shifts later. The same is observed in the results from other instances, and thus we consider the results in this figure to be representative of the results of the other instances tested. To determine candidate optimal pause locations, we select the pause location that results in the highest $P_{\text {solve }}$ for each instance and pause duration. In Figure 5, we provide boxplots of the instance-wise optimal pause locations for each pause duration. In this figure, the shift in $s_{\mathrm{p}}^{\mathrm{opt}}$ with $t_{\mathrm{p}}$ is clearly seen. Additionally, this figure shows that there are few outliers, and that they only occur at the shortest pause duration. We attribute these to the observation that the improvement in $P_{\text {solve }}$ from short pauses is not always greater than the normal variation between runs. For longer pauses, the improvement is larger, and thus there are no outliers. We select the median value for each group (pause duration) as the optimal pause location, and provide the specific values in Table 1. Additionally, we report the sum of the TTS values corresponding to the candidate optimal schedules to determine which leads to the shortest TTS. We find that a schedule with a $1 \mu \mathrm{s}$ pause at $s_{\mathrm{p}}=0.430$ results in the shortest TTS and therefore we expect the corresponding schedule to lead to the shortest TTS in the second stage of our proposal.

Crafting annealing schedules from the estimated $s_{\mathrm{p}}^{\text {opt }}$ values in Table 1, we simulate the final step of our proposal on our second set of 80 SSP instances. Additionally, we also anneal with a schedule that does not include a pause, in order to establish a baseline and assess how much of an improvement in TTS our method results in.

Figure 6 shows the general performance of each schedule in terms of the fraction of instances that are solved. The baseline schedule with no pause solves $18.75 \%$ of instances, leaving the majority unsolved. This matches results with pauses that are far from the optimal pause location, as can be seen in Figure 4. However, using the schedules produced by our proposal substantially improves the number of instances solved, and schedules that include a pause duration of $1 \mu \mathrm{s}$ or greater result in every instance being solved. These results show that our method is able to successfully estimate the optimal pause location for a given pause duration.
Table 2: Speedup over baseline schedule

\begin{tabular}{cc}
\hline$t_{\mathrm{p}}(\mu \mathrm{s})$ & Speedup \\
\hline 0.1 & 1.3 \\
0.3 & 2.1 \\
0.6 & 3.0 \\
1.0 & 3.4 \\
5.0 & 2.9 \\
\hline
\end{tabular}

The main goal of our proposal is to achieve an improvement in TTS through pausing, and due to the metric's dependence on $t_{\mathrm{t}}$, the results thus far do not necessarily indicate that the goal is met. We next calculate TTS as described in Step 3 of our proposal. Figure 7 shows the results for each schedule type. Our results show that every schedule produced using the proposed method is able to achieve a lower TTS (improvement) than the baseline schedule without a pause. This is not necessarily in disagreement with existing work [15], as our proposal results in schedules customized for each specific pause duration rather than evaluating multiple pause durations at the same pause location. The speedup that each schedule provides over the baseline is given in Table 2. We find that the optimal schedule is one containing a $1 \mu$ s pause at $s_{\mathrm{p}}=0.430$. Longer pauses than this result in diminishing returns, and thus TTS increases. Additionally, the effectiveness of the first stage of our method is confirmed by the results of the second phase, which show that the true optimal schedule matches the estimated one. Therefore, we consider our method to be effective at estimating an optimal schedule for a class of problems and reducing TTS.

\section{RELATED WORK}

Since quantum annealing devices have become available, various studies have been conducted to evaluate their performance and to develop methods by which it can be improved.

Hen et al. [17] compare quantum annealing and various classical algorithms on problems with properties typical of constraint satisfaction problems and while no clear speedup with quantum annealing is observed, the authors make the case for additional testing on harder problems. Results from Katzgraber et al. [21] also showed no clear speedup and urge testing on tailored problems with future devices that are larger and have reduced noise. One type of problem of particular interest is based on the weak-strong cluster model, a problem designed to exploit quantum tunneling and for which a speedup over classical algorithms has been observed [13]. While quantum annealing does perform better than, and in some cases scales better than, some classical algorithms, specialized algorithms still outperform quantum annealing. The current state of quantum annealing for optimization is best summarized by Crosson et al. [11]; quantum annealing shows advantages over a select set of classical algorithms, but fails to achieve a scaling advantage over the state-of-the-art classical methods. These results indicate that for current quantum annealing devices to be competitive over a range of applications and algorithms, additional methods such as annealing schedule tuning or hybrid methods may be needed.

One factor that is common in many studies that focus on finding quantum speedup is the presence of native benchmark problems. 


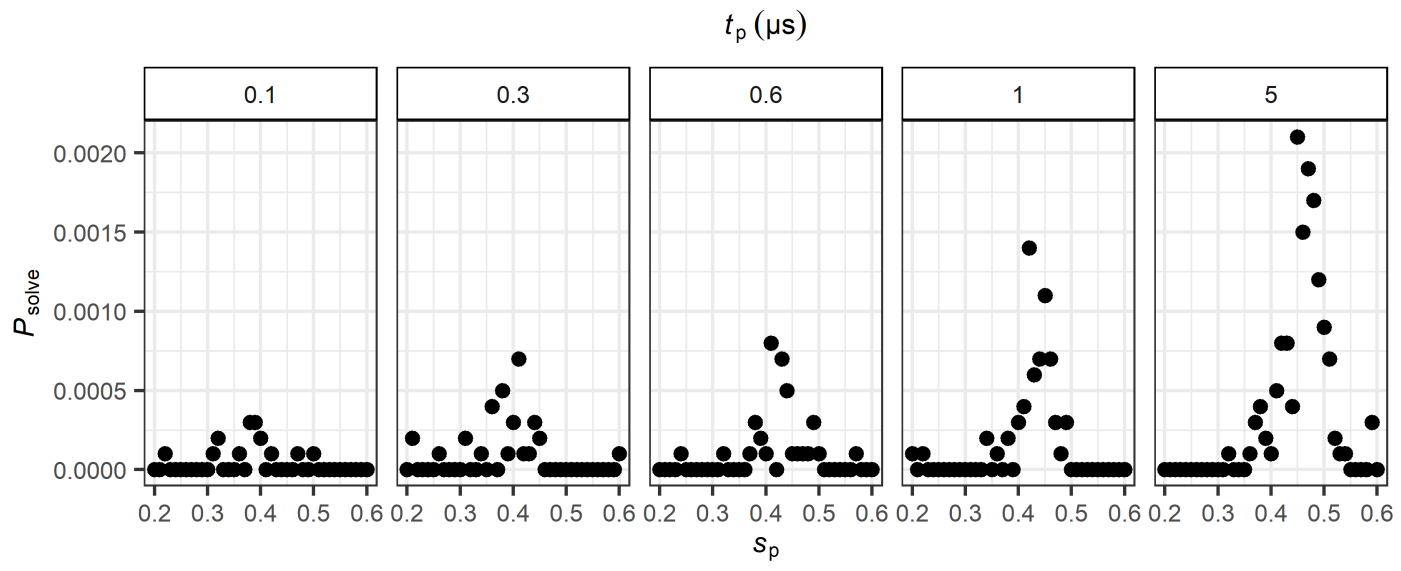

Figure 4: Results for annealing with a pause for various values of $s_{\mathrm{p}}$ and $t_{\mathrm{p}}$. The data shown is from one SSP instance, but representative of other instances.

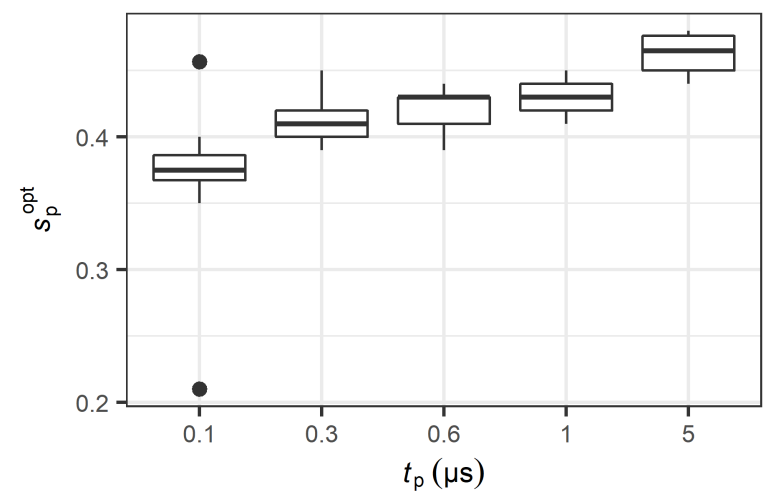

Figure 5: Boxplots of instance-wise optimal pause locations for the pause durations tested.

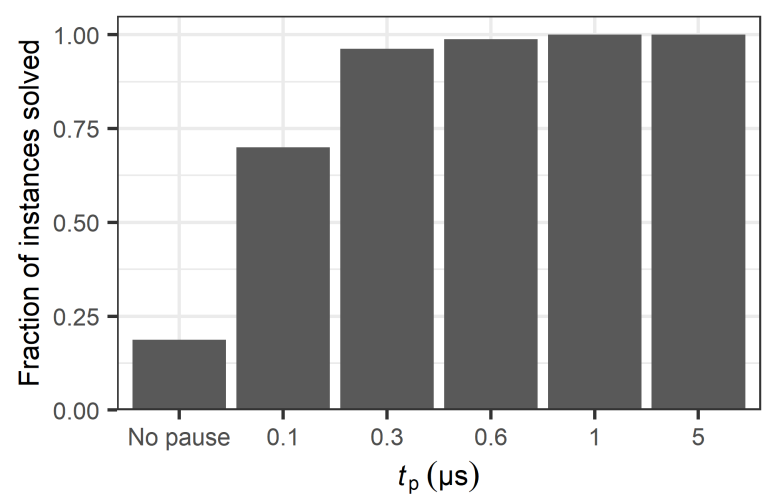

Figure 6: The fraction of the second set of 80 SSP instances that were solved using annealing schedules produced by the proposal.

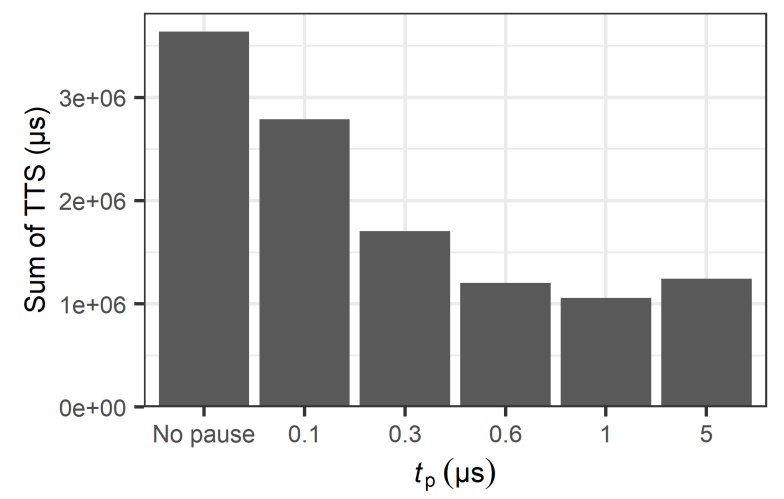

Figure 7: The sum of TTS values for each schedule type.

As the structures of these problems match the physical layout of the annealers, chains of physical qubits are unnecessary. However, many problems of practical interest have arbitrary structure, and thus must undergo embedding. Lucas [24] provides formulations for many fundamental problems, enabling work on more complex applications. Rieffel et al. [27] show the impacts of problem to QUBO formulations as well as embedding on planning problems and emphasize the importance of efficient formulations and hardware connectivity. Other works show the impacts of techniques used in formulation and embedding properties, such as the large qubit overhead required by techniques such as one-hot encoding [33], or the detrimental effects associated with embeddings containing long chains [34]. Date et al. [12] address the challenges of embedding by designing an embedding algorithm that produces smaller embeddings that result in higher quality solutions in less time than the most commonly used embedding algorithm [9]. While the latest generation quantum annealing devices have higher qubit connectivity [7], which reduces the number of qubits required for problems 
with dense connectivity, problem embedding and formulation remain important considerations for achieving high performance with quantum annealing.

Quantum-classical hybrid methods are a collection of approaches that are used to address the shortcomings of current quantum annealing devices. One common approach is using a decomposition algorithm to partition a problem into subproblems that are solved on different solvers. For example, a subproblem with continuous variables is solved with classical resources while a subproblem of discrete variables is optimized with quantum annealing [30, 31] One benefit of problem decomposition is that the subproblems are often smaller, which means that problems of sizes that are too large to be solved by quantum annealing alone can be solved after decomposition. The hybrid approach presented by Abbott et al. [4] recognizes similarities between subproblems and identifies that embeddings can be reused, and thus avoids repeating the costly process of finding an embedding. Another method iteratively reduces the variables to be optimized based on results from previous anneals, and achieves a higher $P_{\text {solve }}$ than repeated annealing without the method[20,29]. These results show that quantum computers can be enhanced by creating algorithms that leverage existing classical techniques.

The introduction of annealing controls in the latest generations of D-Wave devices has prompted many studies on how these controls affect performance. One control, anneal offsets, allows modifications of $A(s)$ and $B(s)$ on a per-qubit basis. Multiple studies have shown that anneal offsets can be used to increase the minimum gap of a system and achieve higher performance than using default values [5, 23]. Another modification to the schedule that is available is reverse annealing, in which the annealer starts in a classical state, introduces quantum fluctuations, and then reduces them. Empirical results show that reverse annealing can improve performance of quantum annealing, but often requires an initial configuration that is close to the optimal configuration [14, 16, 32]. Yet another schedule modification is pausing, which introduces a period in which $A(s)$ and $B(s)$ remain constant. Marshall et al. [25] observed orders of magnitude improvement in the probability of successfully finding the ground state for native problems when a pause was placed in an optimal location. Other works have shows that pausing is beneficial for embedded problems as well $[15,36]$. One challenge in using these controls in practice is that there is a large configuration space; reverse annealing requires a high quality solution to the optimization problem to be solved, and pauses are only effective within a specific region of the anneal that is typically unknown. Yarkoni et al. [35] address this issue by using an evolutionary strategy to tune anneal offsets to values that outperform default values. There are few works with similar goals, and therefore more research on efficiently using annealing controls in practice is necessary.

The main objective of this work is to present a method that uses pausing to achieve a reduction in TTS. While other works have shown that pauses can reduce TTS, one distinguishing characteristic of our work is that we present the steps used to find the pause duration and location for crafting an annealing schedule that outperforms one without a pause. A second characteristic that sets our work apart from others is that we design our method for realistic applications. That is, where real-time tuning of the annealing schedule must be avoided in order to reduce TTS. The relevant related work either does not present methods for tuning annealing schedules with a pause, or does not propose methods that are applicable to real-world applications.

\section{CONCLUSIONS AND FUTURE WORK}

We proposed a method for achieving an improvement in TTS through pausing, over a baseline schedule without a pause. We target problems that can be decomposed into multiple similar problems, or problems that may be repeatedly sampled, so that a single class-wise optimal schedule can be found once and reused. By performing estimation of such a schedule in an offline manner, our proposal avoids the costly process of tuning schedules in real-time. Our evaluation results show that not only does our proposal accurately determine an optimal schedule, but it also results in a 3.4 times speedup over a schedule that does not contain a pause. Being that our method can be employed in real-time without any deterioration to TTS, we consider our proposal to be promising for practical use.

One of the primary limitations of our method is that it targets problems that can be decomposed into multiple similarly structured sub-problems. While this requirement is not unrealistic, there are certainly many applications that do not fit this requirement. A second limitation is that there is no guidance on selecting values for pause duration and location when estimating an optimal schedule. While all of the pause durations tested in this work were able to provide a speedup when optimally placed, this may not be the case for other problem types or pause durations. Increasing the number of schedules tested may lead to more effective schedules being found, but requires additional time and resources. A final limitation is the transferability of an optimal schedule to other problem classes, and similarly, the effectiveness of an optimal schedule on problems that may not be members of the target problem class, but similar nonetheless. For example, we have shown positive results on SSP instances generated from the parameters $N, I_{i}$, and $C$ presented in Section 4 . The optimal schedule found in this work may be effective for SSP instances generated using other parameter values, but also exhibit limited effectiveness, or be ineffective altogether, for other instances. Despite these limitations, this work has shown that our method is effective at improving $P_{\text {solve }}$ and reducing TTS for the SSP.

There are multiple future directions for this work and field. An extension of this work that is able to estimate the shift in the optimal pause location based on the pause duration would be useful as it would reduce the number of anneals required to estimate an optimal schedule. Additionally, due to the success of this method and the optimistic results from other work on annealing schedule modifications, adapting the proposal to reverse annealing or other annealing controls should be explored. One specific potential direction is the combination of the current proposal, which produces high quality solutions through pausing, and methods such as the one in [16], which requires high quality initial configurations for reverse annealing. Another area of interest is methods that are able to approximate the minimum gap of a system in a quick and efficient manner, as this would be valuable for optimizing annealing schedules. Finally, while our method is effective for the SSP instances we 
consider, there may be SSP parameters that generate instances for which the optimal schedule in this work is ineffective. Additional work on determining the similarity between problems is needed to determine if an annealing schedule can be effectively reused. In general, more research on tuning annealing schedules is needed due to limited time that annealing controls have been available. We expect that as future generations of annealers bring improved hardware, properly tuning annealing controls and schedules will be key to improving $P_{\text {solve }}$ and reducing TTS.

\section{ACKNOWLEDGMENTS}

This work is partially supported by MEXT Next Generation HighPerformance Computing Infrastructures and Applications R\&D Program "R\&D of A Quantum-Annealing-Assisted Next Generation HPC Infrastructure and its Applications."

\section{REFERENCES}

[1] [n.d.]. dwave-system. https://github.com/dwavesystems/dwave-system

[2] [n.d.]. QPU Specific Physical Properties. https://support.dwavesys.com/hc/enus/articles/360005268633-QPU-Specific-Physical-Properties

[3] 2020. Practical Quantum Computing: An Update. Retrieved July 16, 2020 from https://www.dwavesys.com/sites/default/files/14-1047A-A_Practical Quantum_Computing_An_Update_0.pdf

[4] Alastair A. Abbott, Cristian S. Calude, Michael J. Dinneen, et al. 2019 A hybrid quantum-classical paradigm to mitigate embedding costs in quantum annealing. International fournal of Quantum Information 17, 05 (2019), 1950042. https://doi.org/10.1142/S0219749919500424 arXiv:https://doi.org/10.1142/S0219749919500424

[5] Juan I Adame and Peter L McMahon. 2020. Inhomogeneous driving in quantum annealers can result in orders-of-magnitude improvements in performance. Quantum Science and Technology 5, 3 (jun 2020), 035011. https://doi.org/10.1088/20589565/ab935a

[6] Sergio Boixo, Troels F. Rønnow, Sergei V. Isakov, Zhihui Wang, David Wecker Daniel A. Lidar, John M. Martinis, and Matthias Troyer. 2014. Evidence for quantum annealing with more than one hundred qubits. Nature Physics 10, 3 (2014), 218-224.

[7] Kelly Boothby, Paul Bunyk, Jack Raymond, and Aidan Roy. 2019. Nextgeneration topology of D-Wave quantum processors. (2019). Retrieved June 21, 2021 from https://www.dwavesys.com/sites/default/files/14-1026A-C_NextGeneration-Topology-of-DW-Quantum-Processors.pdf

[8] Paul I. Bunyk, Emile M. Hoskinson, Mark W. Johnson, Elena Tolkacheva, Fabio Altomare, Andrew J. Berkley, Richard Harris, Jeremy P. Hilton, Trevor Lanting, Anthony J. Przybysz, and Jed Whittaker. 2014. Architectural Considerations in the Design of a Superconducting Quantum Annealing Processor. IEEE Transactions on Applied Superconductivity 24, 4 (2014), 1-10. https://doi.org/10.1109/TASC. 2014.2318294

[9] Jun Cai, William G. Macready, and Aidan Roy. 2014. A practical heuristic for finding graph minors. arXiv:1406.2741 [quant-ph]

[10] Alberto Caprara, Hans Kellerer, and Ulrich Pferschy. 2000. The multiple subset sum problem. SIAM fournal on Optimization 11, 2 (2000), 308-319.

[11] E. J. Crosson and D. A. Lidar. 2021. Prospects for quantum enhancement with diabatic quantum annealing. Nature Reviews Physics 3, 7 (July 2021), 466-489. https://doi.org/10.1038/s42254-021-00313-6

[12] Prasanna Date, Robert Patton, Catherine Schuman, and Thomas Potok. 2019. Efficiently embedding QUBO problems on adiabatic quantum computers. Quantum Information Processing 18, 4 (March 2019). https://doi.org/10.1007/s11128-0192236-3

[13] Vasil S. Denchev, Sergio Boixo, Sergei V. Isakov, Nan Ding, Ryan Babbush, Vadim Smelyanskiy, John Martinis, and Hartmut Neven. 2016. What is the Computational Value of Finite-Range Tunneling? Phys. Rev. X 6 (Aug 2016), 031015. Issue 3. https://doi.org/10.1103/PhysRevX.6.031015

[14] John Golden and Daniel O'Malley. 2021. Reverse annealing for nonnegative/binary matrix factorization. PLOS ONE 16, 1 (01 2021), 1-10. https: //doi.org/10.1371/journal.pone.0244026

[15] Zoe Gonzalez Izquierdo, Shon Grabbe, Stuart Hadfield, Jeffrey Marshall, Zhihui Wang, and Eleanor Rieffel. 2021. Ferromagnetically Shifting the Power of Pausing. Phys. Rev. Applied 15 (Apr 2021), 044013. Issue 4. https://doi.org/10.1103/ PhysRevApplied.15.044013

[16] Erica Grant, Travis S. Humble, and Benjamin Stump. 2021. Benchmarking Quantum Annealing Controls with Portfolio Optimization. Phys. Rev. Applied 15 (Jan 2021), 014012. Issue 1. https://doi.org/10.1103/PhysRevApplied.15.014012
[17] Itay Hen, Joshua Job, Tameem Albash, Troels F. Rønnow, Matthias Troyer, and Daniel A. Lidar. 2015. Probing for quantum speedup in spin-glass problems with planted solutions. Phys. Rev. A 92 (Oct 2015), 042325. Issue 4. https: //doi.org/10.1103/PhysRevA.92.042325

[18] Kazuki Ikeda, Yuma Nakamura, and Travis S. Humble. 2019. Application of Quantum Annealing to Nurse Scheduling Problem. Scientific Reports 9 (2019).

[19] Tadashi Kadowaki and Hidetoshi Nishimori. 1998. Quantum annealing in the transverse Ising model. Phys. Rev. E 58 (Nov 1998), 5355-5363. Issue 5. https: //doi.org/10.1103/PhysRevE.58.5355

[20] Hamed Karimi and Gili Rosenberg. 2017. Boosting quantum annealer performance via sample persistence. Quantum Inf. Process. 16, 7 (2017), 166. https://doi.org/10.1007/s11128-017-1615-x

[21] Helmut G. Katzgraber, Firas Hamze, Zheng Zhu, Andrew J. Ochoa, and H. MunozBauza. 2015. Seeking Quantum Speedup Through Spin Glasses: The Good, the Bad, and the Ugly. Phys. Rev. X 5 (Sep 2015), 031026. Issue 3. https://doi.org/10. 1103/PhysRevX.5.031026

[22] Vaibhaw Kumar, Gideon Bass, Casey Tomlin, and Joseph Dulny. 2018. Quantum annealing for combinatorial clustering. Quantum Information Processing 17, 2 (2018), 39. https://doi.org/10.1007/s11128-017-1809-2

[23] Trevor Lanting, Andrew D. King, Bram Evert, et al. 2017. Experimental demonstration of perturbative anticrossing mitigation using nonuniform driver Hamiltonians. Phys. Rev. A 96, 4 (2017), 042322. https://doi.org/10.1103/PhysRevA.96.042322

[24] Andrew Lucas. 2014. Ising formulations of many NP problems. Frontiers in Physics 2 (2014), 5. https://doi.org/10.3389/fphy.2014.00005

[25] Jeffrey Marshall, Davide Venturelli, Itay Hen, et al. 2019. Power of Pausing: Advancing Understanding of Thermalization in Experimental Quantum Annealers. Phys. Rev. Applied 11, 4 (2019), 044083. https://doi.org/10.1103/PhysRevApplied. 11.044083

[26] Florian Neukart, Gabriele Compostella, Christian Seidel, David Von Dollen, Sheir Yarkoni, and Bob Parney. 2017. Traffic Flow Optimization Using a Quantum Annealer. Frontiers in ICT 4 (2017), 29. https://doi.org/10.3389/fict.2017.00029

[27] Eleanor G. Rieffel, Davide Venturelli, Bryan O'Gorman, Minh B. Do, Elicia M. Prystay, and Vadim N. Smelyanskiy. 2015. A case study in programming a quantum annealer for hard operational planning problems. Quantum Information Processing 14, 1 (Jan. 2015), 1-36. https://doi.org/10.1007/s11128-014-0892-x

[28] Troels F. Rønnow, Zhihui Wang, Joshua Job, Sergio Boixo, Sergei V. Isakov, David Wecker, John M. Martinis, Daniel A. Lidar, and Matthias Troyer. 2014. Defining and detecting quantum speedup. Science $345, \quad 6195$ (2014), 420-424. https://doi.org/10.1126/science.1252319 arXiv:https://science.sciencemag.org/content/345/6195/420.full.pdf

[29] Gili Rosenberg, Mohammad Vazifeh, Brad Woods, et al. 2016. Building an iterative heuristic solver for a quantum annealer. Comput. Optim. Appl. 65, 3 (2016), 845869. https://doi.org/10.1007/s10589-016-9844-y

[30] Tony T. Tran, Minh Do, Eleanor Gilbert Rieffel, et al. 2016. A Hybrid QuantumClassical Approach to Solving Scheduling Problems. In Proceedings of the Ninth Annual Symposium on Combinatorial Search, SOCS 2016, Tarrytown, NY, USA, fuly 6-8, 2016, Jorge A. Baier and Adi Botea (Eds.). AAAI Press, 98-106. http: //aaai.org/ocs/index.php/SOCS/SOCS16/paper/view/13958

[31] Tony T. Tran, Zhihui Wang, Minh Do, Eleanor Gilbert Rieffel, Jeremy Frank, Bryan O'Gorman, Davide Venturelli, and J. Christopher Beck. 2016. Explorations of Quantum-Classical Approaches to Scheduling a Mars Lander Activity Problem. In Planning for Hybrid Systems, Papers from the 2016 AAAI Workshop, Phoenix, Arizona, USA, February 13, 2016 (AAAI Workshops, Vol. WS-1612), Daniele Magazzeni, Scott Sanner, and Sylvie Thiébaux (Eds.). AAAI Press. http://www.aaai.org/ocs/index.php/WS/AAAIW16/paper/view/12664

[32] Davide Venturelli and Alexei Kondratyev. 2019. Reverse quantum annealing approach to portfolio optimization problems. Quantum Machine Intelligence 1, 1 (May 2019), 17-30. https://doi.org/10.1007/s42484-019-00001-w

[33] Davide Venturelli, Dominic J. J. Marchand, and Galo Rojo. 2016. Quantum Annealing Implementation of Job-Shop Scheduling. In Proceedings of the Eleventh Workshop on Constraint Satisfaction Techniques for Planning and Scheduling, COPLAS 2016, London, UK, Fune 13-14, 2016, Miguel A. Salido and Roman Barták (Eds.). 25-34. https://icaps16.icaps-conference.org/coplas.html

[34] S. Yarkoni, A. Plaat, and T. Back. 2018. First Results Solving Arbitrarily Structured Maximum Independent Set Problems Using Quantum Annealing. In 2018 IEEE Congress on Evolutionary Computation (CEC). 1-6. https://doi.org/10.1109/CEC. 2018.8477865

[35] Sheir Yarkoni, Hao Wang, Aske Plaat, et al. 2017. Boosting Quantum Annealing Performance Using Evolution Strategies for Annealing Offsets Tuning. In Quantum Technology and Optimization Problems - First International Workshop, QTOP@NetSys 2019, Munich, Germany, March 18, 2019, Proceedings (Lecture Notes in Computer Science, Vol. 11413), Sebastian Feld and Claudia Linnhoff-Popien (Eds.). Springer, 157-168. https://doi.org/10.1007/978-3-030-14082-3_14

[36] Michael Zielewski, Mulya Agung, Ryusuke Egawa, and Hiroyuki Takizawa. 2020. Improving Quantum Annealing Performance on Embedded Problems. Supercomputing Frontiers and Innovations 7, 4 (2020), 32-48. https://superfri.org/superfri/ article/view/332 